\title{
Ethnologies
}

\section{Le patrimoine qui attise les conflits}

\section{Itamar Even-Zohar}

Volume 39, numéro 1, 2017

Géopolitique, conflits et patrimoine

Geopolitics, conflicts and heritage

URI : https://id.erudit.org/iderudit/1051063ar

DOI : https://doi.org/10.7202/1051063ar

Aller au sommaire du numéro

\section{Éditeur(s)}

Association Canadienne d'Ethnologie et de Folklore

ISSN

1481-5974 (imprimé)

1708-0401 (numérique)

Découvrir la revue

Citer cet article

Even-Zohar, I. (2017). Le patrimoine qui attise les conflits. Ethnologies, 39(1), 251-264. https://doi.org/10.7202/1051063ar

\section{Résumé de l'article}

Cet article traite des conséquences préjudiciables de certaines pratiques patrimoniales dans les relations de groupes au long de l'histoire humaine. Son objectif est de mettre en évidence les conflits intergroupes qui sont enflammés par le patrimoine. En tant que bien symbolique assurant prestige et avantages à ceux qui le possèdent, le patrimoine a servi depuis des temps immémoriaux à mobiliser la population pour obtenir des gains stratégiques lors des conflits. Il n'est certainement ni la source ni la cause de la plupart des conflits, mais lorsqu'un conflit se déroule, enflammer les sentiments par l'excitation du patrimoine contribue à élever le niveau d'engagement des membres du groupe impliqué. L'utilisation du patrimoine pour attiser les comportements conflictuels provoque évidemment des dommages aux groupes impliqués, mais en même temps évite au patrimoine de perdre complètement son pouvoir de générer ou de maintenir la cohésion socioculturelle.
Ce document est protégé par la loi sur le droit d'auteur. L'utilisation des services d’Érudit (y compris la reproduction) est assujettie à sa politique d'utilisation que vous pouvez consulter en ligne.

https://apropos.erudit.org/fr/usagers/politique-dutilisation/ 


\section{LE PATRIMOINE QUI ATTISE LES CONFLITS}

Itamar Even-Zohar

Université de Tel-Aviv

\section{Introduction}

Le sujet de cet article porte sur les conséquences préjudiciables de certaines pratiques patrimoniales dans les relations de groupes au long de l'histoire humaine ${ }^{1}$. Je sais que ces mots seront désagréables aux oreilles des partisans du patrimoine, mais je crois que c'est notre devoir en tant que chercheurs de ne pas ignorer cet aspect. De plus, cela me paraît nécessaire pour créer un certain équilibre dans les pratiques patrimoniales qui tendent parfois à le convertir en une substitution laïque de la religion. D'un autre côté, sur le plan méthodologique, mon propos est de soutenir que notre analyse du patrimoine doit être préférablement menée dans un contexte plus large que celui qui est souvent pratiqué aujourd'hui, parce que ce qu'on appelle « le patrimoine » n'est qu'un élément - sans doute majeur - dans le faisceau de facteurs qui concourent à la survie de groupes.

\section{La réversibilité du patrimoine}

Inoffensif, le patrimoine?

L'aspect préjudiciable n'est pas passé inaperçu dans les études sur le patrimoine, mais il n'a peut-être pas reçu toute l'attention qu'il mérite, bien que ces dernières années, alors que les actes violents contre le patrimoine matériel se sont multipliés, les manifestations d'inquiétude dans la conscience publique sont devenues plus fréquentes. Habituellement, l'attention générale et académique s'est concentrée avant tout sur ce que le terme connote immédiatement et intuitivement, à savoir quelque chose qui ne peut pas être nuisible, qui unit, qui crée du lien. Ce n'est bien sûr pas

1. Basé sur une conférence prononcée au Colloque Géopolitique, patrimoine et conflits, Université Jean Monnet, Saint-Étienne, 11 et 12 décembre 2017. 
un accident ; les termes, dans de nombreuses langues, ont été délibérément engendrés pour créer de tels sentiments. Il est sans doute amplement justifié d'étudier le rôle constructif que le patrimoine a joué, en raison de sa capacité à rendre possible l'action collective par la création d'une solidarité, d'un horizon symbolique ou d'un sentiment d'appartenance qui permettent la coopération et la cohésion entre des individus partageant le même espace. Cependant, il convient de ne pas oublier que ce qui peut bénéficier à un groupe peut aussi en même temps causer des dommages à un autre. De plus, les dommages peuvent frapper tous les groupes impliqués dans divers modes de désaccords et entretenir ces désaccords plutôt que contribuer à leur résolution. Ces occurrences sont si évidentes que même des institutions patrimoniales bien établies l'ont reconnu malgré elles, bien que pour elles, les conséquences négatives du fait patrimonial apparaissent clairement comme une surprise déplaisante, comme quelque chose d'absolument inattendu.

La plupart des chercheurs qui s'intéressent au patrimoine acceptent aujourd'hui que la transmission intergénérationnelle du répertoire culturel puisse ne pas être considérée en soi comme patrimoine, à moins que les traits transmis ne soient estampillés et marqués. Marquer les traits culturels, qu'ils soient matériels ou immatériels, pour les rendre précieux aux yeux de ceux qui les possèdent, est une pratique connue depuis l'aube de l'histoire. Historiquement parlant, l'ensemble matériel a comporté des traits tels que les bâtiments monumentaux, l'or et les pierres précieuses, les statues et les stèles, les meubles, peintures et textes, chars et chevaux, les jardins suspendus et autres merveilles. L'ensemble immatériel, d'autre part, a consisté en éléments idéationnels tels que le bien-être, la prospérité, la fierté, l'égalité, la démocratie, la loi et l'ordre, et ainsi de suite. Tous ces éléments ont clairement servi à symboliser le pouvoir, l'identité, et à acquérir du prestige - et continuent de le faire. Avoir un patrimoine est donc devenu indispensable pour assumer non seulement une position de premier plan dans le système mondial, mais en fait pour conserver un pouvoir, quelle que soit sa nature. Les peuples ou les groupes qui ont précédé les autres et qui ont accumulé et capitalisé ces traits ont également acquis de meilleures options pour les convertir en actifs, ou les garder comme tels pendant une période prolongée. Ils bénéficient d'une sorte de privilège de l'antériorité, ce qui leur assure une rente symbolique. En revanche, les nouveaux arrivants, tels que les nouveaux groupes ou les nouveaux États et nations, ont dû soit les adopter des groupes antérieurs, soit les inventer. Bien sûr, des circonstances nouvelles peuvent permettre d'ajouter de nouvelles 
composantes à l'ordonnancement patrimonial établi et ainsi d'obtenir de meilleures options pour acquérir de tels actifs.

\section{Le " prestige " comme enjeu de pouvoir}

Une telle compréhension du patrimoine le place immédiatement dans une dynamique de compétition interculturelle pour les ressources (matérielles et immatérielles) où intervient la notion de concours de statut basé sur le prestige. La fonction du prestige consiste très concrètement à avoir de meilleurs accès aux ressources. Évidemment, l'accès aux ressources a toujours été la tâche et la préoccupation principale de tout être vivant sur la terre. Pour le monde animal, l'accès à la nourriture, à l'habitat et au compagnon a toujours compté parmi les ressources les plus élémentaires nécessaires à la survie, et naturellement encore plus dans les situations de pénurie. On admet généralement que c'est essentiellement depuis et à cause de la révolution agricole (qui a généré plus de ressources mais d'un autre côté beaucoup plus de gens qui revendiquent leur part) que la compétition est devenue un facteur majeur à la fois individuel et dans les relations intergroupes.

La compétition pour la maîtrise des ressources conduit inévitablement à des affrontements de diverses échelles, et des groupes humains se sont en effet engagés dans de tels affrontements depuis des temps immémoriaux. Cependant, l'engagement dans des affrontements directs n'est pas la seule modalité utilisée dans cette compétition. Et souvent même ce n'est pas la plus importante comme moyen d'obtenir des gains stratégiques. Il n'a pas fallu longtemps à des groupes humains pour découvrir que la violence directe peut devenir coûteuse en termes de pertes de ressources humaines et matérielles. Il y a bien sûr dans l'histoire des cas de belligérance continue, mais les rapports agonistiques ne sont finalement pas particulièrement bénéfiques aux groupes belligérants s'ils ont recours à la violence continue. Durant la plus grande partie de l'histoire de l'humanité, les affrontements avec belligérance n'ont été qu'intermittents. Les groupes en conflit cherchaient surtout à obtenir un avantage par des relations stables, dans le but ultime de gagner sécurité et prospérité.

Par conséquent, diverses alternatives à la violence directe ont émergé dans l'histoire. La stratégie la plus visible était celle que j'appellerais la violence indirecte. Celle-ci consistait à avertir tous les autres groupes qu'il ne leur serait pas utile ou profitable de s'engager dans une agression. Annoncer aux autres combien de moyens de destruction on possède (qu'il 
s'agisse de chevaux, de guerriers, de chars, ou d'avions et de missiles) est souvent devenu beaucoup plus rentable que d'utiliser en réalité toutes ces ressources. C'est le principe si paradoxal de la dissuasion : il faut créer un sentiment de peur en jouant sur la disproportion des forces. Comme l'ont suggéré Henrich et Gil-White (2001) dans leur étude sur l'évolution du prestige, la peur crée de la déférence envers la partie appréhendée, à savoir une reconnaissance de ses prétentions supérieures.

Évidemment, avec cette stratégie, l'humanité est passée de l'application exclusive de la force à l'application du symbolisme. On dirait aujourd'hui le soft power. Les coûts de cette stratégie sont encore très élevés, car si l'on ne fait que propager le pouvoir sans avoir le support matériel, le symbolisme peut se révéler inopérant. Pour échapper à cette difficulté, on a développé un échelon intermédiaire qui consiste à parvenir à la déférence non pas en créant la peur, mais en gagnant le respect, ce qui s'est avéré beaucoup plus rentable.

\section{Les biens symboliques comme ressources}

C'est simplement parce que le respect crée les conditions d'une possibilité d'agir de façon volontaire et sans contrainte (apparente), ce qui constitue un mode de relations intergroupes soutenable et plus durable. Évidemment, pour obtenir le respect des autres, les signes officiels du pouvoir ne suffisent pas à assurer ce pouvoir. D'autres attributs doivent être affichés à la place, comme la possession d'objets de valeur recherchés. La France, pour prendre un exemple remarquable, a toujours su revendiquer le respect de ces biens culturels. "L'image de la France est indissociable de sa culture : les touristes étrangers le savent, qui se rendent en grand nombre au Louvre ou au Centre Georges-Pompidou et assistent aux représentations de l'Opéra-Bastille ou de la Comédie-Française ${ }^{2}$ ». L'ancien président américain Obama a même surpassé les Français quand, le 22 novembre 2016, lors d'une cérémonie très médiatisée à la Maison Blanche, au cours de laquelle des chanteurs et musiciens, comédiens, philanthropes, sportifs et autres célébrités ont reçu la Presidential Medal of Freedom, il se vantait comme suit : " [a]nd it's useful, when you think about this incredible collection of people, to realize that this is what makes us the greatest nation on Earth. [...] And what a glorious thing that is! What a great gift that is

2. Page Internet publiée en français, anglais et allemand par le ministère des Affaires étrangères de la France lors des premiers jours d'Internet, en septembre 1995. Elle a rapidement disparu peu de temps après. 
to America! ${ }^{3}$ »

Aucune des stratégies que je viens de mentionner n'aurait de sens sans reconnaître qu'elles présupposent non seulement un réseau existant de relations entre groupes, mais aussi l'existence d'un accord sur la valeur des biens qui peuvent être affichés pour gagner en prestige. En pratique, une fois que ces biens deviennent des valeurs convenues, ils deviennent des actifs. Les actifs ne peuvent pas exister sans un marché (Bourdieu 1971), c'est-à-dire sans une bourse. En d'autres termes, les relations intergroupes montrent clairement que si la concurrence a certainement conduit à des affrontements, elle a également conduit à l'établissement de négociations entre les parties et à un système d'échange. L'un des résultats les plus remarquables de ce compromis historique a été la diffusion croissante des répertoires culturels sur de vastes territoires représentant une grande diversité de groupes. On pense au cinéma, par exemple. Plus ces répertoires sont communément partagés, plus il devient évident pour les praticiens de départager ce qui est apprécié de ce qui ne l'est pas pour obtenir un statut.

L'idée de remporter la compétition sur des biens symboliques n'est pas du tout évidente. Nous ne pouvons pas vraiment expliquer comment elle a émergé dans l'histoire, mais nous avons de nombreux aperçus de son fonctionnement depuis l'Antiquité, et j'oserais dire que nous avons des preuves convaincantes non seulement de son existence écrasante, mais aussi de son efficacité. Les preuves deviennent cependant beaucoup plus abondantes au cours des deux derniers siècles et demi, d'abord en Europe et progressivement dans d'autres parties du monde. D'une façon pareille à la situation qui prévalait dans le Croissant fertile depuis l'aube de l'histoire depuis au moins 5000 ans, les entités politiques en compétition en Europe depuis la fin du XVIII e siècle devaient justifier leur légitimité pour acquérir un statut. La concurrence pour gagner en reconnaissance et en légitimité a généré un riche répertoire de biens symboliques qu'une entité se devait de prétendre posséder pour s'imposer. Les divers objets de prestige et de distinction, comme les peintures et les textes, ou les discours et les mythes prétendant à l'ancienneté et à l'antériorité comme gages de supériorité, ont été mobilisés et diffusés par les agents centraux de la planification culturelle à des fins d'affirmation politique et géopolitique.

3. «Et c'est utile, quand on pense à cette incroyable réunion de gens, de réaliser que c'est cela qui fait de nous la plus grande nation de la Terre. [...] Et quelle glorieuse chose! Quel beau cadeau pour l'Amérique! » (Obama 2016) 


\section{Qui possède le passé}

Des querelles au sujet de « qui possède le passé » ont par conséquent surgi dans toutes les régions du monde. Dans ce contexte, l'archéologie (généralement financée et soutenue par les États et autres organismes intéressés) est souvent devenue un outil majeur pour promouvoir des images qui ont nourri un imaginaire symbolique propre à justifier une politique de présence ou de domination. Les efforts laborieux déployés par des groupes de toute taille pour exposer, relater, promouvoir et commercialiser des biens symboliques indiquent au moins une pratique profondément enracinée, et vraisemblablement une conviction profonde que ce type de capital est toujours bénéfique en termes de profits. Alors que, dans l'Antiquité, la rhétorique politique annonçait que les biens appartenaient matériellement aux souverains et seulement métaphoriquement à tous les autres, à l'époque moderne, avec la montée en puissance du nationalisme moderne vers la fin du XVIII e siècle et l'avènement des États, elle a évolué pour justifier et servir un projet d'affirmation identitaire des membres du groupe et de construction d'un sentiment d'appartenance.

L'inculcation délibérée par la propagande directe et les organes éducatifs de plus en plus étendus et contrôlés centralement ont puissamment servi à persuader des populations disparates d'accepter de leur libre volition leur appartenance supposée, c'est-à-dire l'adhésion et la loyauté à une certaine communauté. Le résultat de telles actions peut en effet être ce que nous appelons habituellement la « cohésion » socioculturelle, qu'on peut interpréter en termes contradictoires comme une solidarité humaine, mais en même temps comme une mesure moins coûteuse pour parvenir à l'obéissance collective. Le patrimoine, bien culturel par excellence qui a vocation à faire « communauté », se nationalise et se trouve instrumentalisé à cette fin.

Qui est alors effectivement intéressé par ce type de promotion par des capitaux symboliques ? Sans doute ceux qui décident et contrôlent, c'està-dire les dirigeants et les organes de domination en général. Cependant, on observe, tant dans l'Antiquité qu'aujourd'hui, que le processus d'identification par les membres du groupe avec les biens patrimoniaux proclamés semble croître dans des situations d'affrontements et de conflits, violents ou non. Toutes sortes de querelles, comme celle récente de juillet 2017 sur la question de savoir qui a les droits pour l'ensemble des monuments situés sur la colline sacrée de Jérusalem, peuvent inciter des gens normalement indifférents à prendre tout d'un coup parti dans une querelle qui parfois ne touche pas concrètement leur vie quotidienne. La dimension 
symbolique du patrimoine prend ici tout son sens. Et elle peut déboucher sur du réel, voire encourager la violence dans son sens le plus concret.

\section{Négation, usurpation, appropriation}

\section{La colline sacrée de Jérusalem, exemple parfait}

La colline sacrée de Jérusalem est un exemple parfait (mais c'est un exemple parmi tant d'autres) des diverses stratégies utilisées dans les affrontements pour obtenir l'avantage sur les adversaires. Il s'agit en réalité d'un faisceau de procédures, qui passent de l'annihilation complète et de l'élimination du patrimoine de l'autre à sa négation par l'adoption, par l'usurpation directe, ou par l'appropriation. Annihilation et élimination signifient qu'un groupe détruit le patrimoine d'un autre, à la fois physiquement, politiquement et mentalement. Les méthodes assyriennes et babyloniennes de destruction des villes conquises, la dévastation romaine de Carthage et de Jérusalem ou la destruction par les talibans des statues de Bouddha ne sont que des exemples emblématiques qui ont marqué la conscience collective. De façon identique, la décision de Staline d'inonder le territoire présumé de Sarkel, la ville médiévale de l'Empire khazar, par une nouvelle construction de barrage près d'Astrakhan a été attribuée à son désir d'effacer la mémoire des Khazars, sujet sensible à l'époque soviétique, de façon similaire à ses efforts pour éliminer la mémoire de la Horde d'Or, symbole de la domination sur les Russes, et d'autres événements qui portaient ombrage au roman de l'histoire de la Russie. En effet, Staline interdisait toute recherche sur les Khazars, craignant qu'on puisse arriver à prouver que la Russie descendait d'un «État juif »... Mais il n'était pas le seul à manifester cette aversion émotionnelle proche de l'hystérie ; on la retrouvait dans diverses couches d'une société russe animée de cette crainte d'être privée de son héritage (Shnirelman 2001, 2007 et 2012).

\section{Les moyens d'élimination subtils}

La négation, l'usurpation et l'appropriation peuvent apparaître comme des moyens d'élimination plus subtils, mais en fait ils ne sont pas moins radicaux, et peut-être même plus encore pour la partie affectée. Ces stratégies ne visent pas seulement à éliminer le patrimoine de l'autre à la fois physiquement et dans la mémoire, matériellement et immatériellement : elles ambitionnent d'assumer sa possession à la place de l'autre. Le groupe victorieux ne détruit ni n'efface le patrimoine dans la mémoire, mais au 
contraire l'adopte en se le réappropriant, tout en niant en même temps les droits du propriétaire précédent. Par exemple, les monuments du patrimoine païen (comme les temples et autres lieux de culte) sont transformés en églises, les églises sont converties en mosquées (comme la basilique de Jérusalem ou celle de Sainte-Sophie à Constantinople devenue Istanbul, aujourd'hui un musée), et les synagogues ainsi que les mosquées sont converties en églises, par exemple dans toutes les parties de l'Espagne après l'expulsion des musulmans et des juifs en 1492. Cela s'étend à d'autres types de possessions, comme diverses instances de propriétés intellectuelles. Par exemple, la Bible hébraïque devient le bien des peuples chrétiens, pour ne pas parler des protagonistes hébreux, comme les patriarches et les prophètes, adoptés ou confisqués par diverses autres cultures.

La colline sacrée de Jérusalem, dont les édifices ont été détruits par les Babyloniens et les Romains, a été partiellement reconstruite avec une église byzantine, qui ensuite a été détruite mais finalement reconstruite en 705 de notre ère par le calife al-Walid dans sa forme de basilique pour fonctionner comme une mosquée. Elle est devenue une église avec les croisades après 1099, puis réhabilitée comme une mosquée sous Sallah ad-Dîn (Saladin) en 1187. Les traditions populaires ont présenté la colline comme le lieu où le patriarche Abraham a pris son fils Isaac pour le sacrifier à Dieu. Mais l'islam efface Isaac de la mémoire pour le remplacer par Ismaël. On a même institué une fête pour marquer l'événement dans la mémoire collective, à savoir la Fête du Sacrifice ('īid al-aḍhā ; عيد الأضحى). Il faut noter qu'il ne s'agit pas là d'une tradition arabe préislamique indigène, car même le format du nom montre son origine grecque plutôt qu'arabe ou même hébreu. Il s'agissait certainement, comme l'histoire du vol nocturne de Mohamet vers la colline sur son cheval miraculeux al-Buraq, de réclamer sa possession et en conséquence sa valeur symbolique, et d'en exproprier les autres parties impliquées.

Dans cette guerre de possession, le double jeu d'appropriation et de substitution a joué son rôle dans le traitement des noms. Pendant une longue période, la colline reçut en arabe le nom de Bayt al-Maqdis ( La Maison du Temple »), traduction littérale de l'hébreu Bet ha-Miqdash (בית המקדש), donnant même son nom à la ville entière. Cela fut plus tard abrégé en $\mathrm{Al}$ Quds ( La sainteté »), mais les conflits récents ont conduit les activistes arabes à prendre possession une fois de plus du nom Bayt al-Maqdis pour nommer des organisations et institutions, comme « Le Centre de Jérusalem pour les études documentaires » (مركز بيت المقدس للدراسات التوثيقية). De l'autre côté, le nom actuel de la colline en arabe, «le Noble Sanctuaire » (Al-Haram 
ash-Sharif ; الحرم الشريف), coupe tous les liens avec le nom d'origine.

Ce processus d'usurpation et de réappropriation patrimoniales est toujours actif grâce à la puissance de diffusion d'Internet utilisée par des journalistes, des semi-savants et même des autorités religieuses islamiques, qui vont jusqu'à nier l'existence historique des temples judaïques sur la colline $e^{4}$.

Le complexe entier, qui se trouve maintenant sous domination israélienne mais est administré, selon les accords avec la Jordanie, par le Waqf islamique, est devenu ces dernières décennies de plus en plus un symbole de la résistance contre l'occupation israélienne de la vieille ville de Jérusalem, et une synecdoque pour le rejet de l'existence même de l'État d'Israël sur le territoire de la Palestine créé par les Britanniques en 1918. Une guerre du patrimoine s'est déclenchée, ou plutôt « enflammée » entre les parties en conflit, et de temps en temps ses flammes montent très haut. Après 1967, des groupes messianiques juifs, qui jusque-là acceptaient la domination de l'islam sur la colline, commencèrent à rêver de réhabiliter les anciens temples. Un groupe de fanatiques clandestins fut arrêté en 1984 et condamné à la prison pour avoir projeté de faire exploser l'ensemble du site sacré. Tout récemment, le 20 juillet 2017, une quarantaine de Palestiniens et cinq officiers israéliens ont été blessés dans des affrontements violents qui ont éclaté parce que des Palestiniens enragés sont venus protester contre les détecteurs électroniques installés à l'entrée du site après une attaque meurtrière sur la colline la semaine précédente. Le patrimoine, dans ce cas, ne réunit pas ; il divise, il attise les tensions.

Il y a bien sûr pas mal d'autres disputes patrimoniales en Terre Sainte. Dernier exemple en date : la Grotte des Patriarches à Hébron, considérée comme ayant été à l'origine un temple édomite, puis un édifice monumental hérodien, une église byzantine, et finalement une mosquée. Exceptionnellement, l'UNESCO est intervenue dans le différend par sa décision de juillet 2017 de déposséder Israël de tout droit historique sur le

4. Les arguments à cet égard sont très variés et typiquement contradictoires : on nie l'existence des temples ( « on manque de preuves matérielles»), ou on nie qu'il y ait un lien entre « les juifs d'aujourd'hui » et « les fils de Jacob, les Israélites d'antan ». Un autre argument vise à promouvoir l'idée que Moïse, « le fondateur du judaïsme », n'aurait eu aucun rapport avec Jérusalem, « puisqu'il est né et mort en Égypte » (selon la tradition islamique). Un autre argument avance que, même s'il est vrai qu'il y avait des temples judaïques sur la colline, les Arabes de Palestine sont quand même plus anciens que les juifs, étant « d'origine cananéenne ». 
site 5 . Cela a provoqué quelques déclarations enflammées, notamment du ministère israélien des Affaires étrangères. Un nouveau chapitre de cette saga fut écrit le $1^{\text {er }}$ décembre 2017, quand le musée Bibelhaus de Francfort a annoncé qu'une exposition des manuscrits de la mer Morte n'aurait pas lieu, car le gouvernement allemand ne pouvait pas garantir le retour des manuscrits à l'Autorité des Antiquités d'Israël en raison des revendications palestiniennes et jordaniennes concernant leur propriété. Un nouveau chapitre de cette guerre patrimoniale s'ouvre le 6 décembre 2017, quand le président des États-Unis, Donald Trump, reconnaît Jérusalem comme la capitale d'Israël.

\section{L'enjeu géopolitique du patrimoine}

On voit très bien ici que le patrimoine est mobilisé et instrumentalisé pour gagner une bataille symbolique, mais importante sur le plan géopolitique. Le patrimoine en lui-même n'est certainement ni la source ni la cause de la plupart des conflits, mais lorsqu'un conflit se déroule déjà, même dans des situations où l'arsenal de mesures physiques est efficace entre les mains d'une partie, attiser les sentiments par l'excitation du patrimoine contribue toujours à élever le niveau d'engagement des membres du groupe impliqué. Il semble que le besoin soit encore plus fort et peut-être plus efficace lorsque le groupe concerné est le plus faible dans le conflit et que le symbolisme peut alors devenir le dernier recours en l'absence d'autres moyens. Dans de telles situations, même s'il n'existait pas de ressource patrimoniale disponible pour une telle utilisation, soit en raison de l'efficacité prouvée du patrimoine, soit parce qu'il a déjà été établi dans des circonstances historiques analogues, les groupes n'ont pas de difficulté à inventer sur-le-champ des répertoires patrimoniaux et prétendre qu'ils sont anciens. Si tel est effectivement le cas, j'ose dire que si quelqu'un songe à la disparition utopique du patrimoine, les réalités montrent qu'il peut fonctionner même s'il n'existe pas encore.

Mais des cas comme celui de Jérusalem (dont le nom signifie ironiquement « la ville de la paix ») font parfois songer à ce que pourrait être une disparition des patrimoines ! Certains en viennent à penser que la disparition de ce prétexte belligène pourrait peut-être améliorer la qualité de

5. Le vendredi 7 juillet 2017, le Comité de l'UNESCO inscrit sur la liste «zone protégée » du patrimoine mondial la vieille ville d'Hébron. Douze membres du Comité réuni à Cracovie ont voté pour l'inscription, six se sont abstenus et trois ont voté contre. 
vie des gens plutôt que les engager dans des conflits qui ne mènent nulle part.

Cette utilisation du patrimoine dans les situations de conflit aide paradoxalement le patrimoine (et évidemment ses adhérents) à conserver son potentiel ou son pouvoir réel à une époque où il semble l'avoir perdu. Au moins dans les pays occidentaux, jusqu'à récemment, à savoir avant les nouvelles vagues d'immigration et les menaces terroristes, il semblait que les gens étaient devenus assez indifférents au patrimoine. Il est largement reconnu qu'il devenait de moins en moins un outil d'organisation socioculturel et de plus en plus une marchandise susceptible de générer des revenus, surtout en attirant des gens exogènes au groupe pour le consommer de diverses manières, ce qui dans la plupart des cas est simplement exprimé par le tourisme. L'utilisation du patrimoine pour encourager les comportements conflictuels cause évidemment des dommages aux groupes impliqués, mais en même temps évite au patrimoine de complètement perdre son pouvoir de générer ou de maintenir la cohésion.

Bien que mon objectif premier dans cet article ait été de mettre en évidence les conflits intergroupes attisés par le patrimoine, il faut aussi mentionner les conflits intragroupes comme un cas où le patrimoine est mêlé. Je fais référence à des situations où les manières de vivre des gens ordinaires sont menacées non pas par un ennemi extérieur, mais par les instances dirigeantes, souvent démocratiquement élues. L'un de ces cas est le préjudice causé aux habitants de nombreux quartiers urbains par les autorités qui décident d'évacuer les résidents afin de préserver ce que certains chercheurs appellent " la patrimonialisation » (heritageisation, Hammami 2015 ; Harvey 2001 ; Smith 2006), à savoir l'utilisation du patrimoine disponible ou fabriqué afin de générer des conditions de profits financiers au détriment des populations locales. Selon Hammami et Uzer (2017),

[...] authorities select specific places and objects, and place value on them through processes of "heritageisation" [...]. Such processes often provide authorities with legitimate and moral reason to intervene in people's daily lives [...], and construct the historic and cultural values of places and objects. This may develop into enforced urban change and result in "displacement" (Lees, Bang Shin, and López-Morales 2015), "gentrification" (Non 2016), "exclusion” (Ingram 2016), "marginalisation" (Wacquant 2007), "spatial cleansing" (Herzfeld 2006), or "alienation" of both built environment and community (Timothy and Guelke 2008). (Hammami et Uzer 2017 : 1) 
Dans ce type d'affrontements entre le patrimoine imposé d'en haut et le sentiment du patrimoine local ( "d'en bas »), nous voyons non seulement comment le patrimoine cause des dommages aux citadins modernes, mais aussi comment il a probablement pu le faire dans les diverses périodes du passé, même les plus reculées, au temps où des édifices tels que les pyramides soumettaient les gens à des conditions de vie pénibles. Dans ces cas, ce qui est engagé, c'est un patrimoine contre un autre, à savoir le patrimoine officiel, souvent fabriqué, contre le patrimoine de la vie quotidienne des gens, exprimé également par des traits nettement marqués, mais qui ne rapporte pas de bénéfices du point de vue, soit de la concurrence entre groupes, soit de la commercialisation. C'est un aspect du patrimoine qui devrait être pris en compte dans toute analyse des aspects destructifs du patrimoine.

\section{Conclusion}

Les agences du patrimoine tendent toujours à le présenter comme une composante indispensable de toute culture, qui performe des fonctions utiles et positives pour améliorer la qualité de vie de tout groupe vis-à-vis de tous les autres au travers de l'acquisition de prestige qui a vocation à produire des avantages. Le fait que l'insistance sur la nécessité du patrimoine mène inévitablement à la création et à l'amplification de rivalités est souvent ignoré, ainsi que le fait que ces rivalités génèrent des conflits ayant des résultats préjudiciables pour toutes les parties engagées. Il est temps pour les recherches sur le patrimoine de jeter un œil critique sur ce complexe, d'admettre ses conséquences dangereuses et d'en rendre compte. 


\section{Références}

Bourdieu, Pierre, 1971, «Le marché des biens symboliques ». L'Année sociologique $22: 49-126$.

Hammami, Feras, 2015, «Conservation, Innovation and Healing of the Well-Preserved Medieval Ystad ». Journal of Urban Research and Practice $8(2): 165-195$.

Hammami, Feras et Evren Uzer, 2017, « Heritage and resistance: irregularities, temporalities and cumulative impact ». International Journal of Heritage Studies 24 (5) : 1-20.

Harvey, David C., 2001, « Heritage Pasts and Heritage Presents: Temporality, Meaning and the Scope of Heritage Studies ». International Journal of Heritage Studies 7 (4) : 319-338.

, 2015, « Heritage and Scale: Settings, Boundaries and Relations ». International Journal of Heritage Studies 21 (6) : 577-593.

Harvey, David, 2012. Rebel Cities: From the Right to the City to the Urban Revolution. Londres, Verso.

Henrich, Joseph et Francisco J. Gil-White, 2001, "The evolution of prestige: freely conferred deference as a mechanism for enhancing the benefits of cultural transmission ». Evolution and Human Behavior 22 (3) : 165-196.

Herzfeld, Michael, 2009, Evicted from Eternity: The Restructuring of Modern Rome. Chicago, The University of Chicago Press.

Ingram, Mark, 2016, «Emplacement and the Politics of Heritage in LowIncome Neighbourhoods of Marseille ». International Journal of Heritage Studies 22 (2) : 117-130.

Lees, Loretta, Hyun Bang Shin et Ernesto López-Morales (dir.), 2015, Global Gentrifications. Uneven Development and Displacement. Bristol, Policy Press.

Obama, Barack, 2016, «Remarks by the President at Presentation of the Presidential Medal of Freedom, November 22, 2016 ». The White House, Office of the Press Secretary, en ligne (https://www.whitehouse. gov/the-press-office/2016/11/22/remarks-president-presentationpresidential-medal-freedom).

Shnirelman, Victor A., 2001, «Aryans and Khazars ». Dans John K. Roth et al. (dir.), Remembering for the Future: The Holocaust in an Age of Genocide, Londres, Palgrave Macmillan UK : 884-896.

-, 2007, "The story of a euphemism: The Khazars in Russian nationalist literature ». Dans Peter B. Golden, Haggai Ben-Shammai et András Róna-Tas (dir.), The world of the Khazars: New perspectives, Leyde et Boston, Brill : 353-372. 
-, 2012, Хазарский миф: идеология политического радикализма

в России и ее истоки. Jérusalem et Moscou, Гешарим et Мосты культуры.

Smith, Laurajane, 2006, Uses of Heritage. Londres, Routledge.

Timothy, Dallen et Jeanne Kay Guelke, 2008, Geography and Genealogy:

Locating Personal Pasts. Hampshire, Ashgate Publishing Ltd.

Wacquant, Loïc, 2007, « Territorial Stigmatization in the Age of Advanced Marginality ». Thesis Eleven 91 (1) : 66-77. 